\title{
The importance of omega-3 fatty acids for behaviour, cognition and mood
}

\author{
Alexandra J. Richardson \\ University Laboratory of Physiology, Oxford, UK
}

Abstract

There is mounting evidence that functional deficiencies or imbalances in certain highly unsaturated fatty acids (HUFA) of the omega-3 and omega-6 series may contribute to a wide range of developmental and psychiatric conditions, including dyslexia, dyspraxia, attention deficit hyperactivity disorder (ADHD), autism, depression, bipolar disorder and the schizophrenia spectrum. These nutrients are essential to the development and function of the brain, but the omega-3 HUFA in particular (eicosapentaenoic and docosahexaenoic acids) are often lacking from modern diets. The evidence implicating omega-3 fatty acids in these conditions is summarized here, with a focus on the results from randomized controlled treatment trials. These indicate that treatment with eicosapentaenoic acid can reduce symptoms in adults with mood disorders, schizophrenia and possibly Huntington's disease. In children, preliminary evidence suggests that omega-3 HUFA could also reduce some of the behavioural and learning difficulties associated with ADHD, dyslexia and related conditions. The key features of most of these conditions can be found in milder form as part of normal individual differences in mood, behaviour and cognition. The evidence presented here therefore has implications for the general population, and indicates that an adequate dietary intake of omega-3 fatty acids in particular may be crucial for optimal mental health and functioning.

Keywords: brain; development disorders; mental health; unsaturated fatty acids

\section{Introduction}

The childhood developmental conditions of attention deficit hyperactivity disorder (ADHD), dyslexia, dyspraxia and autistic spectrum disorders are each defined by a different, and relatively specific, pattern of difficulties in behaviour and/or learning. In ADHD these involve attentional problems and/ or hyperactive-impulsive behaviours; in dyslexia, specific difficulties with reading and writing; in dyspraxia, specific weaknesses in the organization, planning and coordination of actions, and in the autistic spectrum, impaired social and communication skills. Between them these conditions affect up to $20 \%$ of school-age children, although their boundaries with normal individual variation in behaviour, cognitive and mood are often less than clear-cut. The associated difficulties persist into adulthood, with substantial costs not only to the individuals and families directly affected, but also to society as a whole (1).

A major difficulty with all of these diagnoses is that they are little more than descriptive labels for particular patterns of behaviour. Their causes are almost always multifactorial, and may differ con- siderably between individuals with the same diagnostic label. In practice, the overlaps between these conditions are very high, and they also tend to cluster in the same families, suggesting common elements at the level of biological predisposition. Increasing evidence suggests that these could include a constitutional susceptibility to functional deficiencies in certain highly unsaturated fatty acids (HUFA) (2).

These common developmental conditions also show familial and other associations with certain psychiatric conditions usually identified in adulthood, including depression and bipolar (manicdepressive) disorder as well as schizophrenia spectrum disorders and some personality disorders. Fatty acid abnormalities also appear to play a role in these conditions, and again, the existing evidence indicates that contributory genetic factors may include mild abnormalities of fatty acid metabolism that act to increase the usual dietary requirements for these essential nutrients $(3,4)$.

Only environmental factors, however, could possibly explain the apparent increases over recent years in the incidence and severity of some of these 
conditions, notably depression, ADHD and autistic spectrum disorders. Changes affecting nutrition are probably among these, and there has been a particularly dramatic reduction in the dietary intake of omega-3 fatty acids in most developed countries (5). As the following section makes clear, this has potential implications for many aspects of brain development and function, to which some individuals will be more vulnerable than others.

\section{The importance of highly unsaturated fatty acids for the brain}

Omega- 6 and omega-3 fatty acids are crucial for normal brain structure and function, but must be derived from the diet. Among them, four HUFA are particularly important for the brain: dihomogamma-linolenic acid (DGLA) and arachidonic acid (AA) (omega-6), and eicosapentaenoic acid (EPA) docosahexaenoic acid (DHA) (omega-3) (6, 7). These can usually be synthesized from the corresponding essential fatty acids (EFA), linoleic acid and $\alpha$-linolenic acid, respectively, as shown in Table 1. Dietary provision of both omega- 6 and omega- 3 is essential, as conversion between them is not possible in humans.

Structurally, AA and DHA are key components of neuronal membranes, making up $15-20 \%$ of the brain's dry mass and more than $30 \%$ of the retina. AA is crucial to brain growth, and mild deficiencies are associated with low birth weight and reduced head circumference. It also plays a key role in the cellular processes underlying learning and memory. DHA is particularly concentrated in highly active membranes such as synapses and photoreceptors, and adequate supplies are essential for normal visual and cognitive development (8).

Throughout life, adequate supplies of HUFA remain crucial for optimal brain function (6). They increase the fluidity of neuronal membranes, essential for efficient signal transduction, and certain HUFA (notably AA and EPA) act as second messengers in chemical neurotransmitter systems, as well as contributing to numerous other aspects of cell signalling. Functionally, three HUFA (DGLA, AA and EPA) are particularly important as substrates for the eicosanoids, a large group of highly bioactive hormone-like substances including prostaglandins, leukotrienes and thromboxanes among others. These HUFA derivatives play key roles in regulating blood flow, hormonal systems and immune functioning, and can modulate ion channels, neurotransmitter uptake, synaptic transmission, apoptosis and many other biological processes. They can therefore have profound influences on brain development and function. An adequate supply and an appropriate balance of HUFA are thus required for normal brain function, both during early development and throughout life. Unfortunately, there are many possible reasons why their availability may be less than optimal.

\section{Possible reasons for functional HUFA deficiencies}

First, levels of these fatty acids are low in many modern diets, particularly those in which highly processed foods predominate (5). The omega-3 HUFA that the brain needs (EPA and DHA) are found in appreciable quantities only in oily fish and seafood, while the omega-3 EFA $\alpha$-linolenic acid

Table I. Pathways for the synthesis of omega- 6 and omega- 3 fatty acids

\begin{tabular}{|c|c|c|c|c|}
\hline \multicolumn{2}{|l|}{ Omega-6 series } & \multirow[t]{2}{*}{ Enzymes involved in HUFA synthesis } & \multicolumn{2}{|l|}{ Omega-3 series } \\
\hline Linoleic (LA) & $18: 2$ & & $\alpha$-Linolenic (ALA) & $18: 3$ \\
\hline$\downarrow$ & & Delta 6-desaturase & $\downarrow$ & \\
\hline$\gamma$-Linolenic (GLA) & $18: 3$ & & Octadecatetraenoic & $18: 4$ \\
\hline$\downarrow$ & & Elongase & $\downarrow$ & \\
\hline Dihomogamma-linolenic (DGLA) & $20: 3$ & & Eicosatetraenoic & $20: 4$ \\
\hline$\downarrow$ & & Delta-5-desaturase & $\downarrow$ & \\
\hline Arachidonic (AA) & $20: 4$ & & Eicosapentaenoic (EPA) & $20: 5$ \\
\hline$\downarrow$ & & Elongase & $\downarrow$ & \\
\hline Adrenic & $22: 4$ & & Docosapentaenoic (DPA) & $22: 5$ \\
\hline$\downarrow$ & & Elongase, delta-6-desaturase, $\beta$-oxidation & $\downarrow$ & \\
\hline Docosapentaenoic (DPA) & $22: 5$ & & Docosahexaenoic (DHA) & 22:6 \\
\hline
\end{tabular}

HUFA: highly unsaturated fatty acid. 
(ALA) is found in dark green leafy vegetables and some nuts and seeds, but its conversion to EPA and DHA may be limited, as discussed below. Omega-6 fats are more plentiful, as the EFA linoleic acid (LA) occurs in most vegetable oils and AA is found directly in meat and dairy produce, although poor EFA-HUFA conversion could plausibly compromise levels of the eicosanoid substrate DGLA.

Overall, the ratio of omega- 6 to omega- 3 fats in typical Western diets has increased dramatically over the past century, from approximately 3:1 to almost 20:1, and this has been explicitly linked with increased rates of many disorders of physical as well as mental health (5). A lack of omega-3 leads to imbalances in eicosanoid production, because the prostaglandin, leukotriene and thromboxane derivatives of AA and EPA generally have opposing actions. In particular, AA's derivatives promote inflammation, while those of EPA have anti-inflammatory effects, and abnormal autoimmune reactivity is increasingly implicated in the developmental and psychiatric disorders considered here (2).

Secondly, there may be difficulties in the in vivo synthesis of HUFA from EFA. Recent research has shown this process to be remarkably inefficient in humans (9-11), possibly because it is impeded by a wide range of dietary or lifestyle factors (12). These include a high dietary intake of saturated, hydrogenated or trans fats, lack of the necessary vitamin and mineral cofactors (including zinc, magnesium, manganese, and vitamins $B_{3}, B_{6}$ and $C$ ), heavy use of caffeine, viral infections and high levels of stress. HUFA levels are reliably depleted by alcohol consumption and smoking (13), and EFA-HUFA conversion may be impaired for constitutional reasons, as appears to be the case in atopic eczema (14) and diabetes (15). Males are particularly vulnerable to difficulties in HUFA synthesis (10). Similar constitutional inefficiencies in EFA-HUFA conversion have been proposed in ADHD (16), and could also form part of the predisposition to some of the other conditions considered here.

Constitutional differences in other enzymes involved in fatty acid metabolism could also predispose some individuals to functional HUFA deficiencies. HUFA are constantly replaced and recycled during the normal turnover and remodelling of cell membranes and in the chemical cascades triggered by normal cell signalling processes. Phospholipase $\mathrm{A}_{2}\left(\mathrm{PLA}_{2}\right)$ enzymes remove HUFA from membrane phospholipids, creating free fatty acids and other products that are highly vulnerable to oxidation, and must be rapidly recycled, requiring at least two further enzyme steps (4). Numerous other enzymes are involved in the transport and utilization of fatty acids. Individual differences in their efficiency will affect dietary HUFA requirements, and may influence the risk of some developmental or psychiatric disorders.

\section{Fatty acids in adult psychiatric disorders}

Between countries, rates of major depression, postnatal depression and bipolar disorder all show strong inverse relationships to levels of seafood consumption, a proxy measure of omega-3 fatty acid intake. The dramatic increase in rates of depression over the past century (17) coincides with the relative disappearance of omega-3 fatty acids from the diet. Blood biochemical evidence also supports a link between omega-3 deficiencies and depressive symptoms, and an elevation of the ratio of AA to EPA appears to be particularly characteristic (18). The specific deficiencies of omega-3 relative to omega-6 HUFA found in depression are consistent with the immune system abnormalities associated with this condition, and differences in the efficiency of both $\mathrm{PLA}_{2}$ and AAspecific enzymes could help to explain individual vulnerability (19). It has been pointed out that the efficacy of conventional antidepressant drugs may depend at least in part on their immunomodulatory effects, which are typically anti-inflammatory (20, 21).

Converging evidence therefore suggests that dietary treatment with omega-3 HUFA could be helpful in mood disorders. Pure EPA was found to be highly effective in a case of severe treatment-resistant depression (22, 23), and randomized controlled trials have confirmed that it can significantly reduce symptoms of unipolar depression $(24,25)$. The first controlled trial indicated similar benefits for omega3 treatment in bipolar mood disorder (26); and reductions in both depressive symptoms and aggression have now been reported in women with borderline personality disorder following EPA treatment (27). Antisocial and violent behaviour in young offenders was also reduced dramatically by nutritional treatment in another recent controlled trial, although in this case dietary supplementation involved a broad spectrum of micronutrients, including vitamins and minerals as well as omega-6 and omega-3 fatty acids (28). 
In schizophrenia, abnormalities of fatty acid and phospholipid metabolism have been well documented, and consistent findings include depletion of HUFA in red blood cell membranes, excessive activity of PLA $_{2}$ enzymes, increased lipid peroxidation and nuclear magnetic resonance spectroscopic evidence of increased brain membrane phospholipid turnover (3). The heterogeneity within schizophrenia is substantial, hence such findings cannot be expected to generalize to all patients with this diagnosis. Nonetheless, in controlled trials treatment with omega-3 fatty acids has been found to be effective in reducing both positive symptoms (such as hallucinations and delusions) and negative symptoms (such as anhedonia, inattention or lack of volition), in both medicated and unmedicated patients (29-32). Clinical improvements following treatment with EPA have also been shown to correlate with improved blood fatty acid status, reduced membrane phospholipid turnover and reversal of cerebral atrophy $(33,34)$. Negative results have been reported in only one of five controlled trials to date, and that trial involved an unusually high dose of EPA (35-37).

Fatty acid deficiencies in red cell membranes relate to the movement abnormalities often associated with schizophrenia and neuroleptic treatment (38), as well as to spontaneous dyskinesias in the general population (39). These and other observations led to controlled investigations of a possible role for omega-3 treatment in Huntington's disease, which showed that EPA may also be beneficial in this condition (40). The results of a larger randomized controlled trial to confirm this are now imminent.

\section{Fatty acids in developmental disorders of childhood}

Many features associated with ADHD, dyslexia, dyspraxia and autistic spectrum disorders are consistent with deficiencies or imbalances in HUFA (2). These include the excess of males affected, slightly increased tendencies for pregnancy and birth complications and minor physical anomalies, and an increased frequency of atopic or other autoimmune disorders in affected individuals and their relatives. Fatty acid abnormalities could also help to account for some of the key cognitive and behavioural features of these conditions, such as anomalous visual, motor, attentional or language processing, as well as some of the associated difficulties with mood, appetite or digestion, temperature regulation and sleep (41). Direct evidence from both clinical and experimental studies supports the idea that HUFA depletion may play a role in these overlapping developmental conditions (reviewed in ref. (2)). Thus, physical signs of fatty acid deficiency such as excessive thirst, frequent urination, rough, dull or dry hair and skin and soft or brittle nails have been clearly linked with ADHD, dyslexia and autistic spectrum disorders, as have reduced blood concentrations of HUFA.

With respect to fatty acid treatment in these conditions, anecdotal evidence, case reports and open studies have all suggested possible benefits, but to date there have been few properly controlled treatment studies. The first such trials in ADHD involved evening primrose oil [providing the omega6 fatty acid $\gamma$-linolenic acid (GLA)], but showed little clear benefit $(42,43)$. Emphasis since then has shifted to the omega-3 HUFA, and subsequent randomized controlled trials indicate that supplements containing predominantly fish oil (providing the omega-3 HUFA, EPA and DHA) can reduce behavioural and learning difficulties in both ADHD and dyslexia $(44,45)$. However, supplementation with pure DHA was found to be completely ineffective in ADHD (46), consistent with other evidence that EPA, not DHA, may be the key omega-3 fatty acid in functional disturbances of attention, cognition or mood, as discussed further below. Results from the first controlled trial of fatty acid supplements in dyspraxia are imminent, and although no treatment trials of autistic spectrum subjects have yet been reported, such studies are now underway.

At present, firm evidence for HUFA as a useful treatment for these kinds of developmental conditions therefore remains limited. Further clinical trials are clearly needed, but given the heterogeneity and comorbidity issues, an exclusive focus on current diagnostic labels may not be the best approach. For example, it is becoming clear that many children receiving the ADHD diagnosis may actually have an undiagnosed bipolar mood disorder (47), and although stimulant medications have become the primary treatment for ADHD, these could exacerbate difficulties in such cases. Similarly, it has been shown that stimulants are ineffective in $70 \%$ of ADHD children with comorbid anxiety, and that their negative side-effects may also be more prominent in this subgroup $(48,49)$. 
Given the mounting evidence for omega-3 fatty acid deficiency in mood disorders, treatment studies with omega-3 HUFA in these subsets of "ADHD" children would appear to be well warranted.

\section{Discussion}

Omega-3 fats have been replaced in many modern diets by saturated and artificial fats, and to some extent by omega- 6 fats (5). The latter are also essential to health, but an appropriate balance is required, and relative deficiencies of omega-3 appear to underlie a wide range of physical and mental health conditions that pose increasing problems in developed countries. The exact optimal dietary ratio of omega- 6 to omega-3 fatty acids may vary both between individuals and across conditions (5), but in the USA and Europe, evidence suggests that many if not most people would benefit from increasing their dietary intake of omega-3. For brain function, direct intake of the preformed omega-3 HUFA (EPA and DHA) is likely to be most effective, as EFA-HUFA conversion may be limited.

Of the omega-3 HUFA, research has shown that EPA, not DHA, is more important in mediating improvements in mood, behaviour and cognition in the conditions discussed here. Pure DHA was found to be ineffective in treating both depression and schizophrenia, while pure EPA has shown significant benefits in these conditions $(24,25,30-32)$. Pure DHA was also completely ineffective in reducing ADHD symptoms, while preliminary evidence suggests that supplements containing both EPA and DHA may help to do this $(44,45)$. Given that the brain contains little if any EPA, and an abundance of DHA, this was initially a surprising result. However, while DHA is important in brain structure, EPA appears to play a more important role in brain function. In this, its mechanisms of action still remain to be elucidated, but the eicosanoid derivatives of EPA are key regulators of immune, endocrine and cardiovascular functions. In addition, direct actions of EPA on cyclo-oxygenases, lipoxygenases, phospholipases, acylating systems, ion channels, mitochondria and peroxisome proliferator-activated receptors are currently the focus of investigations across many different fields of study.

At present, the possible role of nutrition is very rarely considered in the diagnosis or management of developmental or psychiatric disorders, but the evidence reviewed here provides some good reasons for this situation to change. It also seems likely that diet could play a role in the prevention of such disorders, although formal studies to address this possibility are still needed. In summary, the particular importance of omega-3 HUFA in so many aspects of behaviour, learning and mood, in addition to their established benefits for cardiovascular and immune function, suggests that restoring higher proportions of these fats in modern diets could have very positive implications for public health.

\section{Acknowledgements}

The author's work in this area is supported by the Dyslexia Research Trust and Mansfield College, Oxford. Further information on this research can be found at www.dyslexic.org.uk

\section{References}

1. Nyden A, Paananen M, Gillberg C. Neuropsychiatric problems among children are significantly underdiagnosed. Intervention programs result in better and less expensive care. Lakartidningen 2000; 97: 5634-41.

2. Richardson AJ, Ross MA. Fatty acid metabolism in neurodevelopmental disorder: a new perspective on associations between attention-deficit/hyperactivity disorder, dyslexia, dyspraxia and the autistic spectrum. Prostaglandins Leukot Essent Fatty Acids 2000; 63: 1-9.

3. Peet M, Glen I, Horrobin DF, eds. Phospholipid spectrum disorder in psychiatry. Carnforth: Marius Press; 1999.

4. Bennett CN, Horrobin DF. Gene targets related to phospholipid and fatty acid metabolism in schizophrenia and other psychiatric disorders: an update. Prostaglandins Leukot Essent Fatty Acids 2000; 63: 47-59.

5. Simopoulos AP. The importance of the ratio of omega6/omega-3 essential fatty acids. Biomed Pharmacother 2002; 56: 365-79.

6. Yehuda S, Rabinovitz S, Mostofsky DI. Essential fatty acids are mediators of brain biochemistry and cognitive functions. J Neurosci Res 1999; 56: 565-70.

7. Bourre JM, Dumont O, Piciotti M, et al. Essentiality of omega 3 fatty acids for brain structure and function. World Rev Nutr Diet 1991; 66: 103-17.

8. Uauy R, Hoffman DR, Peirano P, et al. Essential fatty acids in visual and brain development. Lipids 2001; 36: 885-95.

9. Pawlosky RJ, Hibbeln JR, Novotny JA, Salem N Jr. Physiological compartmental analysis of alpha-linolenic acid metabolism in adult humans. J Lipid Res 2001; 42: 1257-65.

10. Burdge GC, Jones AE, Wootton SA. Eicosapentaenoic and docosapentaenoic acids are the principal products of alpha-linolenic acid metabolism in young men. Br $\mathbf{J}$ Nutr 2002; 88: 355-63. 
11. Burdge GC, Wootton SA. Conversion of alpha-linolenic acid to eicosapentaenoic, docosapentaenoic and docosahexaenoic acids in young women. Br J Nutr 2002; 88(4): 411-20.

12. Brenner RR. Nutritional and hormonal factors influencing desaturation of essential fatty acids. Prog Lipid Res 1981; 20: 41-7.

13. Simon JA, Fong J, Bernert JT Jr, Browner WS. Relation of smoking and alcohol consumption to serum fatty acids. Am J Epidemiol 1996; 144: 325-34.

14. Horrobin DF. Essential fatty acid metabolism and its modification in atopic eczema. Am J Clin Nutr 2000; 71(Suppl 1): 367S-72S.

15. Horrobin DF. The roles of essential fatty acids in the development of diabetic neuropathy and other complications of diabetes mellitus. Prostaglandins Leukot Essent Fatty Acids 1988; 31: 181-97.

16. Colquhoun I, Bunday S. A lack of essential fatty acids as a possible cause of hyperactivity in children. Med Hypotheses 1981; 7: 673-9.

17. Cross-National Collaborative Group. The changing rate of major depression. Cross-national comparisons. JAMA 1992; 268: 3098-105.

18. Maes M, Smith R, Christophe A, et al. Fatty acid composition in major depression: decreased omega 3 fractions in cholesteryl esters and increased C20:4 omega 6/C20:5 omega 3 ratio in cholesteryl esters and phospholipids. J Affect Disord 1996; 38: 35-46.

19. Horrobin DF. Phospholipid metabolism and depression: the possible roles of phospholipase A2 and coenzyme Aindependent transacylase. Hum Psychopharmacol 2001; 16: 45-52.

20. Leonard BE. The immune system, depression and the action of antidepressants. Prog Neuropsychopharmacol Biol Psychiatry 2001; 25: 767-80.

21. Kenis G, Maes M. Effects of antidepressants on the production of cytokines. Int $\mathbf{J}$ Neuropsychopharmacol 2002; 5: 401-12.

22. Puri BK, Counsell SJ, Hamilton G, et al. Eicosapentaenoic acid in treatment-resistant depression associated with symptom remission, structural brain changes and reduced neuronal phospholipid turnover. Int $\mathrm{J}$ Clin Pract 2001; 55: 560-3.

23. Puri BK, Counsell SJ, Richardson AJ, Horrobin DF. Eicosapentaenoic acid in treatment-resistant depression. Arch Gen Psychiatry 2002; 59: 91-2.

24. Nemets B, Stahl Z, Belmaker RH. Addition of omega-3 fatty acid to maintenance medication treatment for recurrent unipolar depressive disorder. Am J Psychiatry 2002; 159: 477-9.

25. Peet M, Horrobin DF. A dose-ranging study of the effects of ethyl-eicosapentaenoate in patients with ongoing depression despite apparently adequate treatment with standard drugs. Arch Gen Psychiatry 2002; 59: 913-9.

26. Stoll AL, Severus WE, Freeman MP, et al. Omega 3 fatty acids in bipolar disorder: a preliminary doubleblind, placebo-controlled trial. Arch Gen Psychiatry 1999; 56: 407-12.
27. Zanarini MC, Frankenburg FR. Omega-3 fatty acid treatment of women with borderline personality disorder: a double-blind, placebo-controlled pilot study. Am J Psychiatry 2003; 160: 167-9.

28. Gesch CB, Hammond SM, Hampson SE, et al. Influence of supplementary vitamins, minerals and essential fatty acids on the antisocial behaviour of young adult prisoners. Randomised, placebo-controlled trial. $\mathrm{Br} \mathrm{J}$ Psychiatry 2002; 181: 22-8.

29. Puri BK, Richardson AJ. Sustained remission of positive and negative symptoms of schizophrenia following treatment with eicosapentaenoic acid. Arch Gen Psychiatry 1998; 55: 188-9.

30. Peet M, Brind J, Ramchand CN, et al. Two double-blind placebo-controlled pilot studies of eicosapentaenoic acid in the treatment of schizophrenia. Schizophr Res 2001; 49: $243-51$.

31. Peet M, Horrobin DF, E-E Multicentre Study Group. A dose-ranging exploratory study of the effects of ethyl-eicosapentaenoate in patients with persistent schizophrenic symptoms. J Psychiatr Res 2002; 36: 718.

32. Emsley R, Myburgh C, Oosthuizen P, vanRensburg SJ. Randomized, placebo-controlled study of ethyl-eicosapentaenoic acid as supplemental treatment in schizophrenia. Am J Psychiatry 2002; 159: 1596-8.

33. Puri BK, Richardson AJ, Horrobin DF, et al. Eicosapentaenoic acid treatment in schizophrenia associated with symptom remission, normalisation of blood fatty acids, reduced neuronal membrane phospholipid turnover and structural brain changes. Int J Clin Pract 2000; 54: $57-63$.

34. Richardson AJ, Easton T, Puri BK. Red cell and plasma fatty acid changes accompanying symptom remission in a patient with schizophrenia treated with eicosapentaenoic acid. Eur Neuropsychopharmacol 2000; 10: 18993.

35. Fenton WS, Dickerson F, Boronow J, et al. A placebocontrolled trial of omega-3 fatty acid (ethyl eicosapentaenoic acid) supplementation for residual symptoms and cognitive impairment in schizophrenia. Am J Psychiatry 2001; 158: 2071-4.

36. Horrobin DF. Omega-3 fatty acid in schizophrenia. Am J Psychiatry 2003; 160: 188-9.

37. Fenton WS, Dickerson F, Boronow J, et al. Dr Fenton and colleagues reply. Am J Psychiatry 2003; 160: 189.

38. Vaddadi K. Dyskinesias and their treatment with essential fatty acids: a review. Prostaglandins Leukot Essent Fatty Acids 1996; 55: 89-94.

39. Nilsson A, Horrobin DF, Rosengren A, et al. Essential fatty acids and abnormal involuntary movements in the general male population: a study of men born in 1933 . Prostaglandins Leukot Essent Fatty Acids 1996; 55: 837.

40. Puri BK, Bydder GM, Counsell SJ, et al. MRI and neuropsychological improvement in Huntington disease following ethyl-EPA treatment. Neuroreport 2002; 13: 123-6. 
41. Richardson AJ, Puri BK. The potential role of fatty acids in attention-deficit/hyperactivity disorder. Prostaglandins Leukot Essent Fatty Acids 2000; 63: 79-87.

42. Aman MG, Mitchell EA, Turbott SH. The effects of essential fatty acid supplementation by Efamol in hyperactive children. J Abnorm Child Psychol 1987; 15: 75-90.

43. Arnold LE, Kleykamp D, Votolato NA, et al. Gammalinolenic acid for attention-deficit hyperactivity disorder: placebo-controlled comparison to D-amphetamine. Biol Psychiatry 1989; 25: 222-8.

44. Burgess JR. Attention deficit hyperactivity disorder: observational and interventional studies. In: NIH workshop on omega-3 essential fatty acids and psychiatric disorders, 2-3 September 1998. Bethesda, MD: National Institutes of Health; 1998.

45. Richardson AJ, Puri BK. A randomized double-blind, placebo-controlled study of the effects of supplementation with highly unsaturated fatty acids on ADHDrelated symptoms in children with specific learning difficulties. Prog Neuropsychopharmacol Biol Psychiatry 2002; 26: 233-9.
46. Voigt RG, Llorente AM, Jensen CL, et al. A randomized, double-blind, placebo-controlled trial of docosahexaenoic acid supplementation in children with attention-deficit/hyperactivity disorder. J Pediatr 2001; 139: 189-96.

47. Spencer TJ, Biederman J, Wozniak J, et al. Parsing pediatric bipolar disorder from its associated comorbidity with the disruptive behavior disorders. Biol Psychiatry 2001; 49: 1062-70.

48. Pliszka SR. Effect of anxiety on cognition, behavior, and stimulant response in ADHD. J Am Acad Child Adolesc Psychiatry 1989; 28: 882-7.

49. DuPaul GJ, Barkley RA, McMurray MB. Response of children with ADHD to methylphenidate: interaction with internalizing symptoms. J Am Acad Child Adolesc Psychiatry 1994; 33: 894-903.

\footnotetext{
Dr A. J. Richardson

University Laboratory of Physiology

Parks Road

Oxford OXI 3PT, UK

E-mail: alex.richardson@physiol.ox.ac.uk
} 